\title{
Manuscript title Microparticles Containing MiR-625- $5 p$ In Coronary Artery Reduce Myocardial Fibrosis After Myocardial Infarction
}

\section{Ningxin Wen}

Peking University Third Hospital

Qi Zhang

Peking University Health Science Centre

\section{Xuan Wu}

Peking University Third Hospital

Jianing Gao

Peking University Health Science Centre

Yangkai Xu

Peking University Health Science Centre

\section{Zongzhe Jiang}

Peking University Health Science Centre

Changjie Liu

Peking University Health Science Centre

\section{Liang Ji}

Peking University Health Science Centre

Linyun Zu ( $\nabla$ dr_zly@126.com )

Peking University Third Hospital

\section{Research Article}

Keywords: thrombus aspiration, microparticles, cardiac fibrosis, microRNA

Posted Date: May 28th, 2021

DOl: https://doi.org/10.21203/rs.3.rs-527418/v1

License: (c) (i) This work is licensed under a Creative Commons Attribution 4.0 International License.

Read Full License 


\section{Abstract}

\section{Purpose}

Blood from infarct-related arteries obtained by thrombus aspiration is good material for studying the local microenvironment of blood vessels in myocardial infarction. Here, we aimed to observe the effects of intracoronary microparticles (MPs) on cardiac fibrosis and to find associated microRNAs in MPs.

\section{Methods}

Blood samples were collected from patients with ST-segment elevation myocardial infarction who underwent emergency percutaneous coronary intervention, and sub-supersonic centrifugation was used to separate the MPs.

Results

We found that rats treated with intracoronary MPs showed better cardiac function after myocardial infarction compared with rats treated with PBS control or peripheral MPs. RNA microarray analysis indicated that microRNAs, especially miR-625-5p, may play a role in the process. Supplementation with miR-625-5p inhibited proliferation of cardiac fibroblasts and myocardial fibrosis in a mouse myocardial infarction model.

\section{Conclusion}

Our findings indicate that plasma MPs in infarct-related arteries in patients with acute myocardial infarction can inhibit myocardial fibrosis and improve cardiac function, with a process mediated by miR625-5p and HMGA1 (high mobility group AT-hook 1). The current study may provide a possible reference for thrombus aspiration standard.

\section{Introduction}

Morbidity and mortality related to cardiovascular diseases are increasing steadily in China due to lifestyle changes and an aging population. Cardiovascular diseases, which account for more than $40 \%$ of deaths, have become the leading cause of death in China [8]. Myocardial fibrosis after acute ST-segment elevation myocardial infarction (STEMI) is an important cause of heart remodelling and eventually development of heart failure [4], severely affecting patients' quality of life and long-term prognosis. Excessive myocardial fibrosis after myocardial infarction (MI) leads to a gradual increase in myocardial stiffness, decreased compliance, and decreased myocardial oxygen supply. Therefore, finding ways to inhibit cardiac fibrosis is important to improving the long-term prognosis of patients with MI.

The use of thrombus aspiration is intended to reduce the incidence of distal embolization, achieve early reperfusion, and improve patients' near-term and long-term survival. The blood collected during thrombus aspiration can provide microenvironmental information about the patient's coronary arteries and 
myocardium after MI. However, large-scale clinical trial results $[12,14]$ have shown that conventional thrombus aspiration does not reduce the incidence of major adverse cardiac events or severe heart failure in recent [3] or long-term [6]. One possible explanation is that some beneficial components in infarctrelated arteries, as a compensatory mechanism, are discarded when the thrombus is aspirated. And the loss of this type of myocardial protection may lead to the observed limited clinical benefit from thrombus aspiration.

Extracellular vesicles (EVs) contain a variety of molecules such as proteins and microRNAs (miRNAs), bind to the target cells through a ligand/receptor mode, and directly fuse with cells to transfer active substances. EVs are widely involved in cell adhesion, migration, proliferation, apoptosis, and immune response [10]. In recent years, the physiological and pathological effects of EVs have been gradually recognized. Various states such as inflammatory reaction, cardiovascular disease, tumor, and infection can stimulate excessive production of EVs, and EVs may be considered biomarkers in many diseases. Therefore, we wanted to investigate whether EVs are involved in cardiac repair after MI. EVs are classified as exosomes, microparticles (MPs) or apoptotic bodies according to particle size. Exosomes are generated by exocytosis of multivesicular bodies and are 30 to $100 \mathrm{~nm}$ in diameter, whereas MPs (50$1000 \mathrm{~nm}$ in diameter) are generated by budding from the plasma membrane and are generally larger than exosomes[11]. In this study, we focused primarily on the function of MPs, as the other two classes of EVs had been well studied.

Here, using an animal model, we showed that MPs from the coronary arteries of STEMI patients could inhibit myocardial fibrosis after MI, leading to better cardiac function. Moreover, our RNA microarray analysis indicated that miR-625-5p carried in these MPs inhibits fibroblasts from participating in cardiac fibrosis, through the high mobility group AT-hook 1 (HMGA1) and transforming growth factor $\beta 1$ (TGF- $\beta 1$ ) related pathway. Our findings demonstrate that MPs in the microenvironment may be a cardioprotective factor following MI and indicate that miRNAs play important regulatory roles in cardiac fibrosis.

\section{Material And Methods}

Patients

In this study, we selected STEMI patients who underwent primary PCI in Peking University Third Hospital within 12 hours of an MI from June 2014 to June 2017. Coronary angiography was performed according to the standard Judkins method for femoral artery or radial artery to identify infarct-related artery. According to angiography, patients with heavy thrombus burden (TIMI thrombosis grade $\geq 2$ ) [3] are expected to have thrombus aspiration before stenting. STEMI patients were selected before emergency percutaneous coronary intervention and provided informed consent. If their condition later did not warrant thrombus aspiration, consent was withdrawn). The diagnosis of STEMI complied with the criteria of the 2010 Guidelines for the Diagnosis and Treatment of Acute ST Segment Elevation Myocardial Infarction by the Chinese Medical Association Cardiovascular Society [6]. Exclusion criteria were as follows: 1) patients with acute and chronic infection, liver and kidney dysfunction, blood system diseases, severe 
bleeding, autoimmune diseases, malignant tumors, severe trauma, or acute MI caused by nonatherosclerosis; 2) patients who took antiplatelet drugs for more than 1 week before onset of $\mathrm{Ml}$; 3) nonST-segment elevation Ml; or 4) previous old MI. For every patient, the thrombus is usually aspirated 2 to 4 times with 10 to $15 \mathrm{~mL}$ of blood sample collected, and 10 to $15 \mathrm{~mL}$ of peripheral blood (from the radial or femoral artery puncture site) was collected as a control. This clinical research study was approved by the Clinical Trial Ethics Committee of Peking University Third Hospital [Ethics File Number: PUTH-REC-SOP06-3.0-A27 (2013) Medical Lun Shen (105-3)]. Informed consent was given by all patients prior to enrollment in the study.

Isolation of MPs

The blood samples described above (from thrombus aspiration and peripheral control samples) were centrifuged at $1600 \mathrm{~g}$ for 10 minutes immediately after collected to obtain plasma. Plasma underwent ultracentrifugation at $100,000 \mathrm{~g}$ for $1 \mathrm{~h}$ to pellet the MPs contained. The pellet was resuspended in PBS for MP preparation. To separate the platelet- endothelial- and leukocyte-derived MPs, we label MPs with APC AnnexinV (biolegend, CA, USA) and FITC anti-CD 41 antibody (abcam), PE anti-human CD144 (biolegend, CA, USA) or PerCP/Cy5.5 anti-human CD45 (biolegend, CA, USA), then perform flow cytometry. To denature the proteins, MPs were cooled to $-170^{\circ} \mathrm{C}$ and heated to $100^{\circ} \mathrm{C} 10$ times[16].

Preparation of rat MI model

A total of 33 male Sprague Dawley rats at 8 weeks of age, weighing 200 to $250 \mathrm{~g}$, were randomly divided into a sham operation group $(n=3)$, a PBS group $(n=9)$, an O-MP group (receiving occlusion microparticles, $n=12$ ), and a C-MP group (receiving peripheral circulation microparticles, $n=9$ ). MPs were from 12 different patients. Rats were anesthetized by intraperitoneal injection of $1 \%$ pentobarbital sodium, placed on a $37^{\circ} \mathrm{C}$ constant temperature heating plate, and then performed tracheal intubation. With the rat under ventilator-assisted breathing, the surgical area was disinfected and a 3-cm transverse incision was made between the third and fourth ribs. Open the 3rd and 4th ribs with a chest opener, and fully expose the heart. Ligate the left anterior descending coronary artery with $6-0$ non-invasive suture threading. The myocardial whitening and weakened pulsation below the ligation site proved a successful MI surgery[7]. After ligation of the left anterior descending coronary artery, $0.1 \mathrm{~mL}$ of PBS (PBS control) or $3 \times 10^{6}$ O-MPs or C-MPs dissolved in $0.1 \mathrm{~mL}$ of PBS was injected directly into the whitened myocardium by one-time injection. After the chest was closed, the rats were returned to spontaneous breathing, tracheal intubation withdrawn, and the ventilator evacuated. Rats in the sham operation group underwent the same process as the three surgery groups except that the left anterior descending artery was not ligated. C57BL/6 Mice also accepted left anterior descending ligation to induce MI. All protocols for animal experiments were approved by the Peking University Institutional Animal Care and Use Committee [LA2018088].

Ultrasound evaluation 
To evaluate cardiac structure and function, echocardiography was performed postoperatively. Four weeks after the operation, the rats underwent basic anesthesia with $4-5 \%$ isoflurane (C3H2CIF5O), and the thoracic body hair was scraped off. Rats were placed in the supine position on a constant-temperature heating plate, and $2.5 \%$ isoflurane was used to maintain anesthesia. Coupling agent was applied to the fixed limbs, and a Vevo770 small animal ultrasound system (Visual Sonics Inc., Toronto, ON, Canada) was used, with a probe frequency of $17.5 \mathrm{MHz}$, detection depth of $2 \mathrm{~cm}$, and scanning speed of 100 $\mathrm{mm} / \mathrm{s}$. Two-dimensional ultrasound showed the left ventricular short-axis view. The left ventricular motion curve was recorded by M-mode ultrasound at the papillary muscle level[7].

Histological analysis

Cardiac sampling and echocardiography were performed within $24 \mathrm{~h}$. For histological analysis, hearts were fixed in $4 \%$ paraformaldehyde $(\mathrm{pH} 7.4)$ for $48 \mathrm{~h}$. The tissue was soaked in paraffin, cut into $6-\mu \mathrm{m}$ sections, and stained with Sirius red or Masson trichrome. Collagen fiber area (Sirius red staining) was analyzed using Image $\mathrm{J}$ software (National Institutes of Health, Bethesda, MD, USA). Quantification of the collagen fiber area was performed by scanning the Sirius red-stained section at 20x magnification using the Leica slice scanning system (Leica Microsystems Inc., US). Image $\mathrm{J}$ was used to quantify the redstained portion and the total area, and the collagen fiber area (red-stained portion) was calculated as a percentage of the total area.

Enzyme linked immunosorbent assay (ELISA)

The contents of TGF- $\beta 1$, connective tissue growth factor (CTGF), collagen (COL)-I, and COL-III (Product No. SEA124, SEA010, SEA571 and SEA176, CLOUD-CLONE CORP., CCC, USA) in the whole heart tissue and fibroblasts were detected by ELISA, according to the kit instructions. The optical density value was measured at $450 \mathrm{~nm} / 579 \mathrm{~nm}$ using a microplate reader.

Cell culture and treatment of cardiac fibroblasts in vitro

Cardiac fibroblasts were from lactating rats and the human cardiac fibroblast (HCF) cell line (Cat. \#6300, Sciencell, California, USA) and were cultured in FM-2 medium. To assess proliferation, cells were seeded in 96-well plates, with 4000 cells/well. Each group set 5 parallel wells, and was cultured at $37^{\circ} \mathrm{C}, 5 \% \mathrm{CO}_{2}$, with $0.1 \mu \mathrm{mol} / \mathrm{L}$ angiotensin II (Ang-II) (Sigma-Aldrich, St. Louis, MO, USA) as a stimulating factor. After $24 \mathrm{~h}$ of dosing, according to the Cell Proliferation ELISA BrdU assay kit instructions (Cat. 11669915001, Roche, Basel, Switzerland), absorbance was measured at $450 \mathrm{~nm}$. As for MTT assay, cells were cultured as same. After treating with $20 \mathrm{ul} /$ well MTT $(5 \mathrm{mg} / \mathrm{ml})$ for 4 hours, dissolve the crystals with $150 \mathrm{ul} /$ well DMSO and measure absorbance at OD $490 \mathrm{~nm}$. Transwell chambers (8- $\mu \mathrm{m}$ pore size) were used to detect cell migration. Cells in each group were collected and resuspended in serum-free Dulbecco's modified Eagle medium, and $150 \mu \mathrm{L}$ of resuspended cells $\left(1 \times 10^{5}\right)$ was added to each upper chamber. The chambers were fixed on 24-well plates. Medium containing Ang-ll was added for stimulation. After $18 \mathrm{~h}$, the chamber was placed under an inverted microscope to observe to observe and count cells in the lower chamber. Counts were subjected to statistical analysis [9]. To access immunofluorescence, cells were 
washed twice with cold PBS before fixing and permeabilizing. Fix the cells with cold 4\% PFA in room temperature for 10 mins and permeabilize with $0.1 \%$ Triton X-100 for 10 mins. F-actin antibody (bioss, Beijing, China), corresponding fluorescent secondary antibody (Thermo Fisher) and DAPI were used to stain cells. Images were obtained using a fluorescence microscope. Images were obtained using a fluorescence microscope.

Western blot

Total protein samples were extracted from cardiac tissues and cardiac fibroblasts using lysis buffer [2, 13]. Membranes were incubated with appropriate primary antibodies: alpha-smooth muscle actin (a-SMA) (ab32575, abcam), COL-I (ab34710, abcam), COL-III (ab184993, abcam), HMGA1 (ab129153, abcam), and $\beta$-actin (abclonal, Wuhan, China).

MicroRNA microarray analysis

The MPs obtained by ultracentrifugation were added to an appropriate amount of Trizol for full lysis, and RNA was extracted after lysis. Samples were labeled using the miRCURY Array Power Labeling kit (Qiagen, New York, US). The reagent was mixed and incubated with the sample at $16^{\circ} \mathrm{C}$ for $1 \mathrm{~h}$ in the dark. The labeling enzyme labeled the Hy3TM or Hy5TM fluorophore with a miRNA to obtain a fluorescent probe that hybridized with the microarray. The fluorescence intensity of the microarray was scanned using an Axon GenePix 4000B microarray scanner (Molecular Devices, CA, USA) and read using GenePix Pro 6.0; the intensity of the green fluorescent signal was calculated by subtracting the background from the mean of the four parallel points on each probe. The miRNA expression levels of 10 samples from 2 groups were obtained by median normalization to obtain data normalized to the same order of magnitude, and standard expression was obtained. Probes with fluorescent intensity $\geq 30.0$ in all samples were retained, median standardization was performed on all microarrays, differentially expressed probes were screened, and differentially expressed miRNAs were clustered. Collect the target genes of miRNAs through miRanda, miRbase and Targetscan.

Effect of miRNA on human cardiac fibroblasts

MiRNA mimic (100 nM, ribobio, Guangzhou, China), miRNA inhibitor (200 nM, ribobio, Guangzhou, China), or HMGA1 short interfering RNA (siRNA) were added to HCFs to verify function. MiRNA agomir $(20 \mu \mathrm{M}$, ribobio, Guangzhou, China) was used in intracardiac injection in mice.

Statistical method

The experimental data are expressed as mean \pm standard deviation (SD) of $n$ independent experiments. Statistical analysis was performed using the Prism 4.0 software system (GraphPad Software Inc., La Jolla, CA, USA). Mean comparisons between two groups were performed by using Student's t-test, and data processing between the groups was analysed by analysis of variance. A P-value $<0.05$ was considered statistically significant. 


\section{Results}

\section{General clinical data of patients}

This study enrolled 30 patients with thrombotic aspiration following acute MI from June 2014 to June 2017. The average age of patients was 56 years, $87 \%$ were male, $48 \%$ had hypertension, $42 \%$ had hyperlipidemia, $30 \%$ had diabetes, $64 \%$ had a history of smoking, and $33 \%$ had a decrease in left ventricular ejection fraction. Overall, $93 \%$ of patients had elevated highly-sensitive troponin T (hsTnT), with an average of $4.77 \pm 3.31 \mathrm{ng} / \mathrm{mL}$ and a median of $4.74 \mathrm{ng} / \mathrm{mL}$. Overall, $87 \%$ of the patients had an increased creatine kinase (CK) level, with an average of $1885.64 \pm 1574.77 \mathrm{U} / \mathrm{L}$ and a median of 1600 $\mathrm{U} / \mathrm{L}$. Moreover, $84 \%$ of patients had an increased creatine kinase isoenzyme (CK-MB), with an average of $182.76 \pm 152.62 \mathrm{U} / \mathrm{L}$ and a median of $180 \mathrm{U} / \mathrm{L}$ (Table 1 ).

Table 1

Comparison of general data of patients. Abbreviation: hsTnT, hypertrophic troponin; $\mathrm{CK}$, creatine kinase; CK-MB, creatine kinase isoenzyme.

\begin{tabular}{|c|c|}
\hline \\
\hline Age, years & $56.36 \pm 14.35(55)$ \\
\hline Gender, Male\% & 87.88 \\
\hline Hypertension, \% & 48.48 \\
\hline Hyperlipoidemia, \% & 42.42 \\
\hline Diabetes Mellitus, \% & 30.30 \\
\hline Smoking, \% & 63.64 \\
\hline hsTNT, mg/ml & $4.77 \pm 3.31(4.74)$ \\
\hline $\mathrm{CK}, \mathrm{U} / \mathrm{L}$ & $1889.64 \pm 1574.77(1600)$ \\
\hline CKMB, U/L & $182.76 \pm 152.62(180)$ \\
\hline High hsTNT (>0.1mg/ml), \% & 93.94 \\
\hline High CK (> 170U/L), \% & 87.88 \\
\hline High CKMB (> 24U/L), \% & 84.85 \\
\hline
\end{tabular}

Flow cytometry showed that the MP content in the coronary artery was significantly higher than that in the peripheral blood. To confirm the source of MPs, we used different antibodies to detect them (PerCP/CY5.5-CD45, phycoerythrin-CD144 and FITC-CD41). The number of leukocyte-derived microparticles (MPs) in the coronary aspiration group was $273.43 \pm 113.38 / \mu \mathrm{L}$, which was higher than that in the peripheral circulation group $(202.86 \pm 73.88 / \mu \mathrm{L})$. The number of platelet-derived MPs in the 
coronary aspiration group was $1789.14 \pm 957.09 / \mu \mathrm{L}$, also higher than that in the peripheral circulation group $(698.76 \pm 475.85 / \mu \mathrm{L})$. However, there was no significant difference in the number of endothelial cell-derived MPs between the two groups $(236.87 \pm 89.96 / \mu \mathrm{L}$ vs. $221.98 \pm 105.67 / \mu \mathrm{L}$; Fig. 1$)$.

\section{Intracoronary MPs improve cardiac function and reduce cardiac fibrosis in rats after MI}

To explore MP function in vivo, 200- to 250-g rats were subjected to MI surgery, with intramyocardial injection with either PBS, occlusion microparticles (O-MPs) from thrombotic aspiration, or peripheral circulation microparticles (C-MPs) $\left(3 \times 10^{6} \mathrm{MPs}\right.$ in $\left.100 \mathrm{ul} \mathrm{PBS}\right)$ at the same time (for sham group, $\mathrm{n}=3$; PBS group, $n=9$; O-MPs group, $n=12 ; C-M P s, n=9$ ). After 28 days, $M$-mode ultrasound showed a higher left ventricular ejection fraction in the O-MP group than in the PBS and C-MP groups (Fig. 2A, B). PicroSirius red staining showed less collagen deposited in the heart of rats in the O-MP group compared with PBS group $(22.4 \% \pm 4.37 \%$ vs. $14.38 \% \pm 2.33 \%)$; no significant difference was found between rats in the PBS group and the C-MP group $(22.4 \% \pm 4.37 \%$ vs. $21.7 \% \pm 5.22 \%)$. Rats in the O-MP group had a lesser degree of left ventricular dilatation (Fig. 2C, D). In cardiac fibrosis after MI, mainly type I and type III collagen are increased, so we detected these two collagens by ELISA in the whole heart tissue of the four groups. The results showed that COL-I was reduced after O-MP treatment $(3.70 \pm 0.37 \mu \mathrm{g} / \mathrm{mL})$ compared with PBS $(6.29 \pm 1.18 \mu \mathrm{g} / \mathrm{mL})$, but not C-MP treatment $(6.28 \pm 0.40 \mu \mathrm{g} / \mathrm{mL})$. There was no significant difference in the content of COL-III between the four groups (Fig. 2E). These findings showed a cardiac protective function of O-MPs, and indicated that myocardial fibrosis is suppressed by O-MPs.

\section{O-MPs inhibit proliferation, migration, and transition of cardiac fibroblasts in vitro}

In this study, we used Ang-II as a stimulating factor for cell proliferation to observe whether O-MPs and CMPs could inhibit the proliferation and transition of cardiac fibroblasts from lactating rats. The MTT assay and BrdU experiment showed the same trend: that proliferation of fibroblasts was significantly inhibited in the O-MP intervention group compared with the Ang-II group (absorbance $1.15 \pm 0.08$ vs. 0.67 $\pm 0.12,0.59 \pm 0.04$ vs. $0.31 \pm 0.09$ ) (Fig. 3A, B). The Transwell experiments showed that O-MP treatment inhibited the migration of cardiac fibroblasts after Ang-ll stimulation ( $243 \pm 17.3$ vs. $141 \pm 21.5)$ (Fig. 3C, D). Western blot showed decreased expression of a-SMA in the O-MP group (Fig. 3E, F), and immunofluorescence staining showed that there was less F-actin and microfilament structure (stained by FITC-labeled phalloidin) in the O-MP group (Fig. 3I). These results indicated that O-MPs inhibited the fibroblast-to-myofibroblast transition. At the same time, western blot results showed that O-MP treatment reduced the expression of COL-I and COL-III induced by Ang-II (Fig. 3G, H), whereas C-MP treatment did not inhibit the fibroblast-to-myofibroblast transition or reduce the expression of collagens. Connective tissue growth factor (CTGF) and transforming growth factor $\beta 1$ (TGF- $\beta 1$ ) are known to play an important role in the process of cardiac fibrosis and can promote the occurrence and development of cardiac fibrosis. Therefore, we observed the effect of MPs on these two pro-fibrotic factors. ELISA showed that levels of CTGF and TGF- $\beta 1$ in heart tissue of rats of other groups were significantly higher than those in the sham operation group and could be inhibited by O-MPs (Fig. 3J). 


\section{MicroRNAs play an important role in 0-MP functions}

MPs contain proteins and microRNAs. To determine whether miRNAs participate in inhibition of fibrosis, O-MPs were cooled to $-170^{\circ} \mathrm{C}$ and heated to $100^{\circ} \mathrm{C} 10$ times to denature the proteins. The MTT assay showed that these miRNAs in O-MPs inhibited the cell viability of cardiac fibroblasts (Fig. 4A). We then performed a microarray analysis of 5 samples each of MPs from the thrombus aspiration group (C group) and the peripheral blood group (W group). Significant Pearson's correlation among biological replicates confirmed reproducibility of the data and the difference between C-MPs and O-MPs (Fig. 4B). The miRNA microarray expression profile showed 307 differentially expressed miRNAs, 221 of which were upregulated and 86 downregulated in the O-MP group (Fig. 4C). Next, we used the miRDB database (http://mirdb.org/) to identify potential miRNAs related to TGF- $\beta$ and other signalling pathways that affect cardiac fibrosis. After comparing these potential miRNAs with the 307 differentially expressed miRNAs, we found 47 miRNAs that might regulate TGF- $\beta 1$ and CTGF (Fig. 4D). Based on the fold changes of these 47 miRNAs, we further screened miR-625-5p and miR-3613-3p.

\section{MiR-625-5p inhibits proliferation of human cardiac fibroblasts (HCFs) via high mobility group AT-hook 1 (HMGA1)}

We treated HCFs with miR-625-5p and miR-3613-3p mimics separately, and both the MTT assay and BrdU experiment showed that miR-625-5p mimic inhibited proliferation of HCFs, but miR-3613-3p mimic did not (Fig. 5A, B). The use of miR-625-5p inhibitor in 0-MP-treated HCFs reduced the effect of O-MPs on HCF proliferation (Fig. 5C). Then we used the Targetscan database (http://targetscan.org) to find potential targets of miR-625-5p, which were KSR2 (kinase suppressor of Ras 2), HMGA1, and APLN (apelin). Real-time PCR showed that transcription of KSR2 and HMGA1 was reduced after treatment with miR-625-5p (Fig. 5D, E, and F). We also found an increased level of HMGA1 after MI (Fig. 5G). Western blot confirmed that miR-625-5p reduced HMGA1 content in HCF (Fig. 5H), and ELISA showed that blocking HMGA 1 by siRNA reduced the level of TGF- $\beta 1$ and CTGF secreted by HCF after Ang-ll induction (Fig. 5I). The results indicated that miR-625-5p may inhibit HCF via HMGA1 and its role in increasing TGF$\beta 1$ and CTGF levels.

\section{MiR-625-5p inhibits myocardial fibrosis after MI in mice}

To verify whether miR-625-5p could inhibit myocardial fibrosis in vivo, 6-week-old c57 male mice (for sham group, $n=6$; NC control group, $n=10$; miR-625-5p agomir group, $n=10$ ) were subjected to $M I$ surgery with intracardiac injection of miR-625-5p agomir ( $20 \mu \mathrm{M}, 25 \mu \mathrm{L}$ each) at the same time. Real-time PCR showed that miR-625-5p levels in the hearts of the injected group were still higher than in controls 14 days after surgery (Fig. 6A). Injection of miR-625-5p reduced mortality in mice after MI (Fig. 6B), and Masson trichrome staining showed a reduction in collagen deposition in the injected group (Fig. 6C). 


\section{Discussion}

Previous studies have found that the peripheral MP level in patients with unstable angina or MI is significantly higher than that of patients with stable angina or non-coronary diseases; no difference is reported in peripheral MP level between patients with stable angina and non-coronary diseases [1]. Here, we further studied MPs in intracoronary blood during acute exacerbation of patients with acute MI to provide a more accurate and direct analysis. We found that the number of MPs in coronary thrombus aspiration blood of patients with STEMI was much higher than that in peripheral blood of the same patients, and the proportion of platelet- and leukocyte-derived MPs in coronary blood was also increased. Ischemia and hypoxia result in a complex environment in infarct-related arteries, and the proportion of cellular components in the infarct site differs from that in peripheral blood. These findings suggest that O-MPs from intracoronary differ in source and quantity from C-MPs, and may reflect the local environment after MI.

In this study, we highlighted the role of O-MPs as a cardioprotective factor. Reduced infarct size was observed in rats receiving O-MPs from MI patients after surgery, with a higher ejection fraction and less collagen deposition. This kind of myocardial protective mechanism could explain part of the reason for the limited clinical benefit of thrombus aspiration. Cardiac fibrosis is an important cause of diastolic dysfunction. A variety of profibrotic cytokines, such as Ang-ll, promote fibroblast proliferation, secretion of collagen, and fibroblast-to- myofibroblast transition, which further prevent recovery of cardiac function [15]. In our study, O-MPs reduced TGF- $\beta 1$, CTGF, and collagen secretion. At the same time, the proliferation, migration, and transition of fibroblasts induced by Ang-II were inhibited by O-MPs, suggesting that the cardioprotective effect of O-MP derives from its anti-cardiac-fibrosis function.

In this study, we found that miRNAs in MPs can inhibit fibrosis. We analysed the miRNA expression profile of MP and its possible downstream targets by gene microarray. We identified 47 differentially expressed miRNAs correlated with cardiovascular disease, many of which have been rarely studied. Some of these, after further research, may have potential as new diagnostic or therapeutic targets for STEMI. Next, we confirmed that hsa-miR-625-5p significantly inhibited the proliferation of cardiac fibroblasts and reduced infarct size in mice after Ml. The similar functions indicate that hsa-miR-625-5p is one of the factors by which intracoronary MPs inhibit cardiac fibrosis. In addition, analysis by Targetscan, RT-PCR, and western blot showed that miR-625-5p may work via HMGA1, a factor that had been shown to promote fibrosis [5].

Due to technical constraints, serum miRNAs may still be present in our MP preparations. We cannot exclude the possible involvement, albeit minor, of a non-particle source of miR-625-5p. In addition, because of the difficulty in obtaining clinical samples and the difference in timing and operation of the percutaneous coronary intervention surgeon's choice of sample, it may take years to accumulate sufficient valid samples, which makes it difficult to completely and accurately quantify compounds in 0MP. 
In summary, we report here that 0-MPs contain functional miR-625-5p that is deliverable to cardiac fibroblast and inhibits its activity (Fig. 6D). We demonstrated that MP and miRNA levels are elevated in MI patients, which represents a cardioprotective mechanism that inhibits myocardial fibrosis. The present study underlines the importance of MPs in the regulation of heart repair after MI, with potential clinical relevance. Accumulation of more samples may lead to further study of the differences between coronary and peripheral MPs and a greater understanding of the potential of MPs as a predictive factor for cardiovascular events.

\section{Declarations}

\section{ACKNOWLEDGEMENTS}

We thank Louise Adam, ELS(D), from Liwen Bianji, Edanz Editing China (www.liwenbianji.cn/ac) for editing the English text of a draft of this manuscript.

\section{Funding}

This work was supported by the National Natural Science Foundation of China [81670323]. Funding for open access charge: National Natural Science Foundation of China.

\section{Code availability}

Not applicable.

\section{Availability of data and material}

Send email to corresponding author for the all details of data and material.

\section{Compliance with Ethical Standards}

\section{Disclosure of potential conflicts of interest}

None.

\section{Research involving Human Participants and/or Animals}

The clinical research study was approved by the Clinical Trial Ethics Committee of Peking University Third Hospital [Ethics File Number: PUTH-REC-SOP-06-3.0-A27 (2013) Medical Lun Shen (105-3)]. All protocols for animal experiments were approved by the Peking University Institutional Animal Care and Use Committee [LA2018088].

\section{Informed consent}

Patients were selected before emergency percutaneous coronary intervention and provided informed consent. If their condition later did not warrant thrombus aspiration, consent was withdrawn). 


\section{References}

1. Biasucci LM, Porto I, Di Vito L, De Maria GL, Leone AM, Tinelli G, Tritarelli A, Di Rocco G, Snider F, Capogrossi MC, Crea F (2012) Differences in microparticle release in patients with acute coronary syndrome and stable angina. Circ J 76:2174-2182 doi:10.1253/circj.cj-12-0068

2. Biro E, Akkerman JW, Hoek FJ, Gorter G, Pronk LM, Sturk A, Nieuwland R (2005) The phospholipid composition and cholesterol content of platelet-derived microparticles: a comparison with platelet membrane fractions. J Thromb Haemost 3:2754-2763 doi:10.1111/j.1538-7836.2005.01646.x

3. Frobert $\mathrm{O}$, Lagerqvist $B$, Olivecrona GK, Omerovic E, Gudnason T, Maeng $M$, Aasa $M$, Angeras $\mathrm{O}$, Calais F, Danielewicz M, Erlinge D, Hellsten L, Jensen U, Johansson AC, Karegren A, Nilsson J, Robertson L, Sandhall L, Sjogren I, Ostlund O, Harnek J, James SK (2013) Thrombus aspiration during ST-segment elevation myocardial infarction. N Engl J Med 369:1587-1597 doi:10.1056/NEJMoa1308789

4. Heusch G, Libby P, Gersh B, Yellon D, Bohm M, Lopaschuk G, Opie L (2014) Cardiovascular remodelling in coronary artery disease and heart failure. Lancet 383:1933-1943 doi:10.1016/s01406736(14)60107-0

5. Hopper RK, Moonen JR, Diebold I, Cao A, Rhodes CJ, Tojais NF, Hennigs JK, Gu M, Wang L, Rabinovitch M (2016) In Pulmonary Arterial Hypertension, Reduced BMPR2 Promotes Endothelial-toMesenchymal Transition via HMGA1 and Its Target Slug. Circulation 133:1783-1794 doi:10.1161/circulationaha.115.020617

6. Lagerqvist B, Frobert O, Olivecrona GK, Gudnason T, Maeng M, Alstrom P, Andersson J, Calais F, Carlsson J, Collste O, Gotberg M, Hardhammar P, loanes D, Kallryd A, Linder R, Lundin A, Odenstedt J, Omerovic E, Puskar V, Todt T, Zelleroth E, Ostlund O, James SK (2014) Outcomes 1 year after thrombus aspiration for myocardial infarction. N Engl J Med 371:1111-1120 doi:10.1056/NEJMoa1405707

7. Lekawanvijit S, Kompa AR, Zhang Y, Wang BH, Kelly DJ, Krum H (2012) Myocardial infarction impairs renal function, induces renal interstitial fibrosis, and increases renal KIM-1 expression: implications for cardiorenal syndrome. Am J Physiol Heart Circ Physiol 302:H1884-1893 doi:10.1152/ajpheart.00967.2011

8. Ma LY, Chen WW, Gao RL, Liu LS, Zhu ML, Wang YJ, Wu ZS, Li HJ, Gu DF, Yang YJ, Zheng Z, Hu SS (2020) China cardiovascular diseases report 2018: an updated summary. J Geriatr Cardiol 17:1-8 doi:10.11909/j.issn.1671-5411.2020.01.001

9. Pluskota E, Woody NM, Szpak D, Ballantyne CM, Soloviev DA, Simon DI, Plow EF (2008) Expression, activation, and function of integrin alphaMbeta2 (Mac-1) on neutrophil-derived microparticles. Blood 112:2327-2335 doi:10.1182/blood-2007-12-127183

10. Porro C, Trotta T, Panaro MA (2015) Microvesicles in the brain: Biomarker, messenger or mediator? J Neuroimmunol 288:70-78 doi:10.1016/j.jneuroim.2015.09.006

11. Raposo G, Stoorvogel W (2013) Extracellular vesicles: exosomes, microvesicles, and friends. J Cell Biol 200:373-383 doi:10.1083/jcb.201211138 
12. Sardella G, Mancone M, Bucciarelli-Ducci C, Agati L, Scardala R, Carbone I, Francone M, Di Roma A, Benedetti G, Conti G, Fedele F (2009) Thrombus aspiration during primary percutaneous coronary intervention improves myocardial reperfusion and reduces infarct size: the EXPIRA (thrombectomy with export catheter in infarct-related artery during primary percutaneous coronary intervention) prospective, randomized trial. J Am Coll Cardiol 53:309-315 doi:10.1016/j.jacc.2008.10.017

13. Sims PJ, Wiedmer T, Esmon CT, Weiss HJ, Shattil SJ (1989) Assembly of the platelet prothrombinase complex is linked to vesiculation of the platelet plasma membrane. Studies in Scott syndrome: an isolated defect in platelet procoagulant activity. J Biol Chem 264:17049-17057

14. Vlaar PJ, Svilaas T, van der Horst IC, Diercks GF, Fokkema ML, de Smet BJ, van den Heuvel AF, Anthonio RL, Jessurun GA, Tan ES, Suurmeijer AJ, Zijlstra F (2008) Cardiac death and reinfarction after 1 year in the Thrombus Aspiration during Percutaneous coronary intervention in Acute myocardial infarction Study (TAPAS): a 1-year follow-up study. Lancet 371:1915-1920 doi:10.1016/s0140-6736(08)60833-8

15. Xia Y, Lee K, Li N, Corbett D, Mendoza L, Frangogiannis NG (2009) Characterization of the inflammatory and fibrotic response in a mouse model of cardiac pressure overload. Histochem Cell Biol 131:471-481 doi:10.1007/s00418-008-0541-5

16. Zhang H, Liu J, Qu D, Wang L, Wong CM, Lau CW, Huang Y, Wang YF, Huang H, Xia Y, Xiang L, Cai Z, Liu P, Wei Y, Yao X, Ma RCW, Huang Y (2018) Serum exosomes mediate delivery of arginase 1 as a novel mechanism for endothelial dysfunction in diabetes. Proc Natl Acad Sci U S A 115:E6927-e6936 doi:10.1073/pnas.1721521115

\section{Figures}



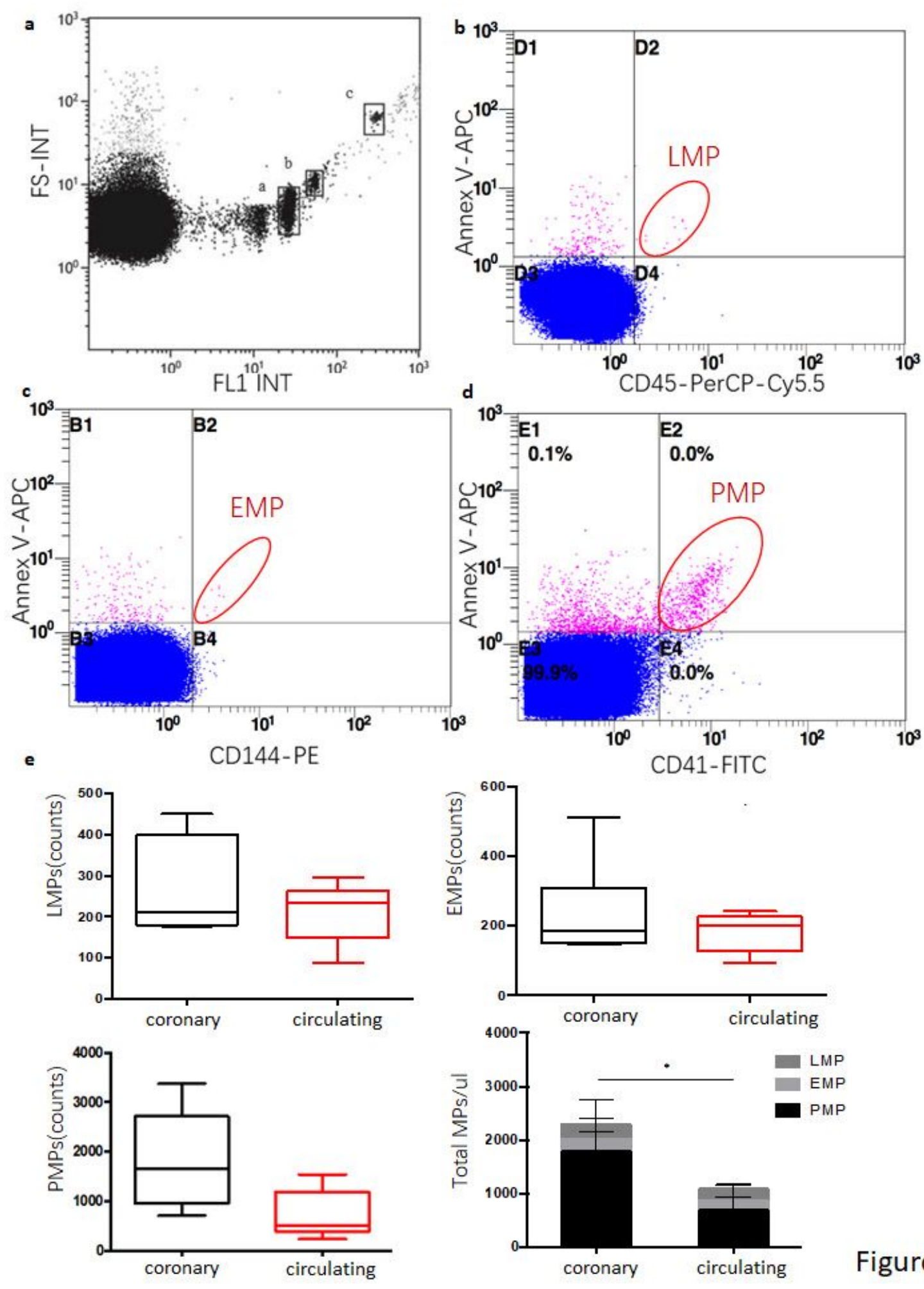

Figure 1

\section{Figure 1}

Flow cytometry to detect particle content and subtypes. (a) Megamix beads (0.5-, 0.9-, and 3- $\mu \mathrm{m}$ premixed beads) were used to determine the gate to measure microparticles (MPs). (b) Leukocyte-derived microparticles (LMPs) were detected by PerCP/CY5.5-CD45 and annexin V; (c) endothelial cell-derived microparticles (EMPs) were identified by phycoerythrin-CD144 markers, and (d) platelet-derived microparticles (PMPs) were identified by FITC-CD41. (e) Compared with the circulating group, the LMPs, 
PMPs, and total number of microparticles in the coronary group were significantly increased; there was no significant difference in EMPs between the two groups. Results are means $\pm S D$. ${ }^{*}<<0.05$ vs. circulating MPs.

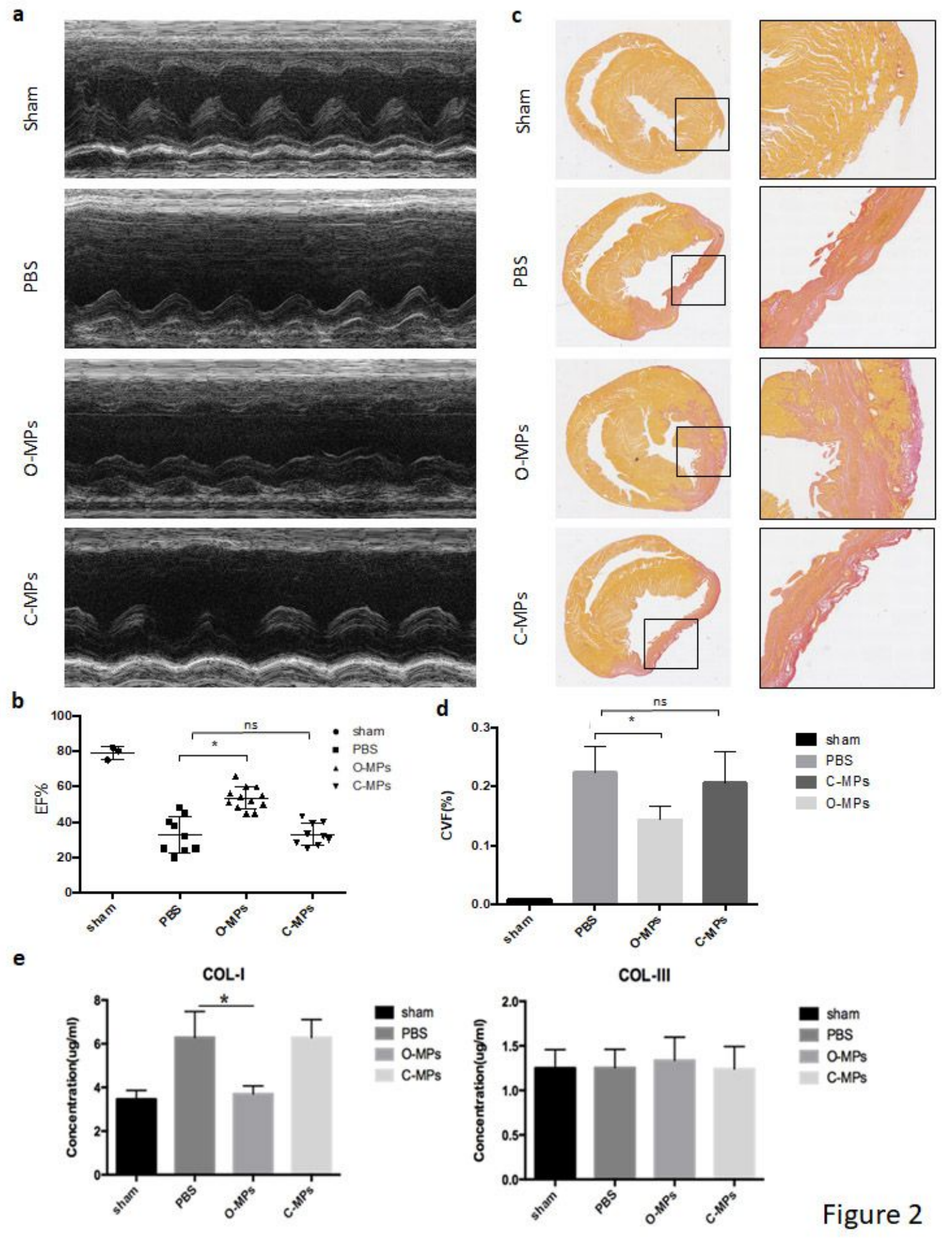

Figure 2

O-MPs improved cardiac function and reduced cardiac fibrosis in rats. Rats were subjected to $\mathrm{Ml}$ as well as intramyocardial injection. (a, b) Ejection fraction, measured by echocardiography. (c) Cardiac fibrosis 
was assessed by Picro-Sirius red staining. (d) Collagen volume fraction of the four groups (sham operation or injection of PBS, O-MPs, or C-MPs). (e) Content of collagens (COL-I and COL-III) in the whole heart tissue by ELISA. $n=3,9,12,9$, in the four groups, respectively. Results are means \pm SD; Student's ttest, ${ }^{*} \mathrm{P}<0.05$.

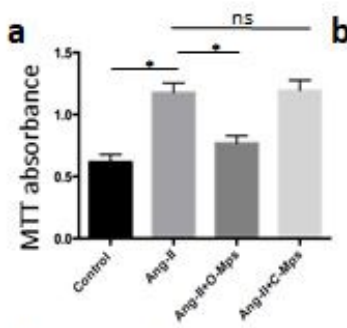

d control

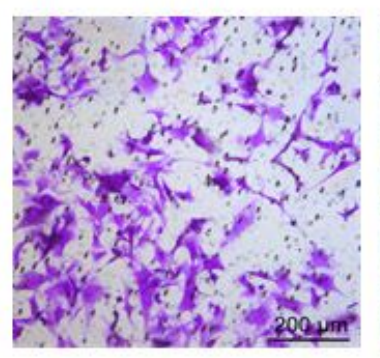

Ang-II + O-MPs
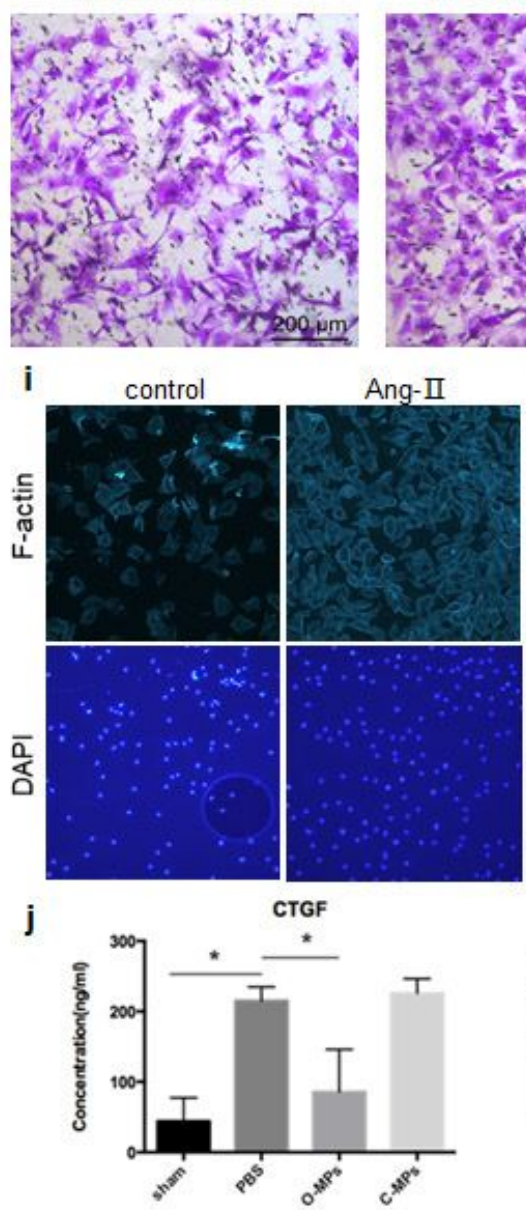

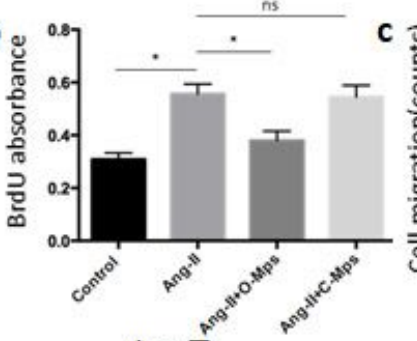

Ang-II

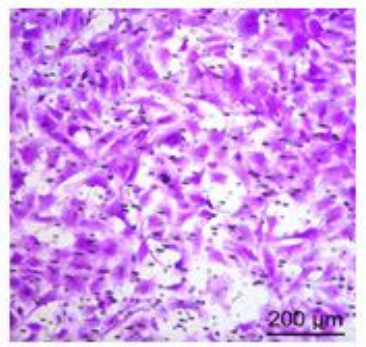

Ang- II + C-MPs

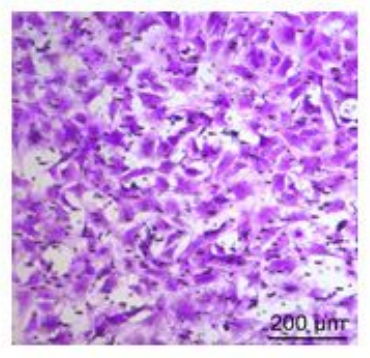

Ang- $\Pi+$ O-MPs
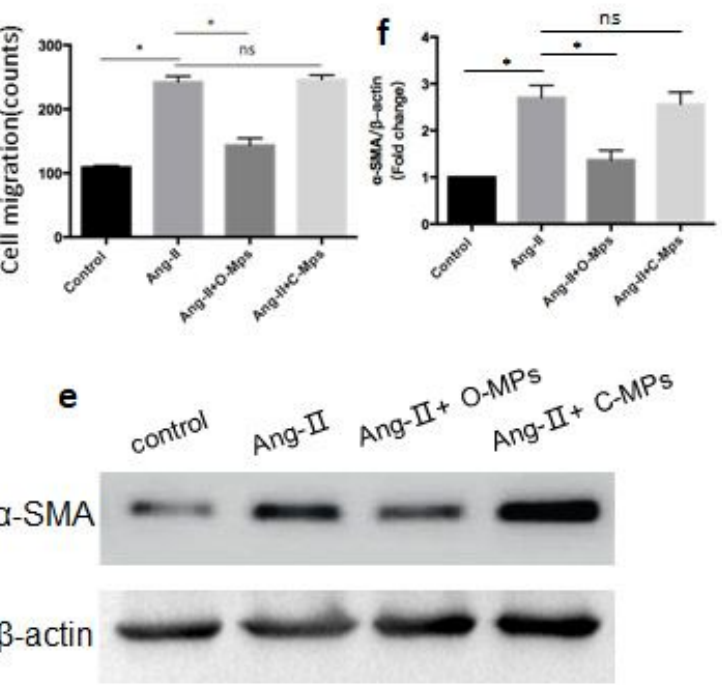

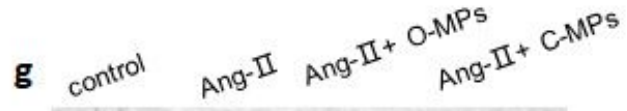

COL-I

COL-III

B-actin

Ang-I + C-MPs
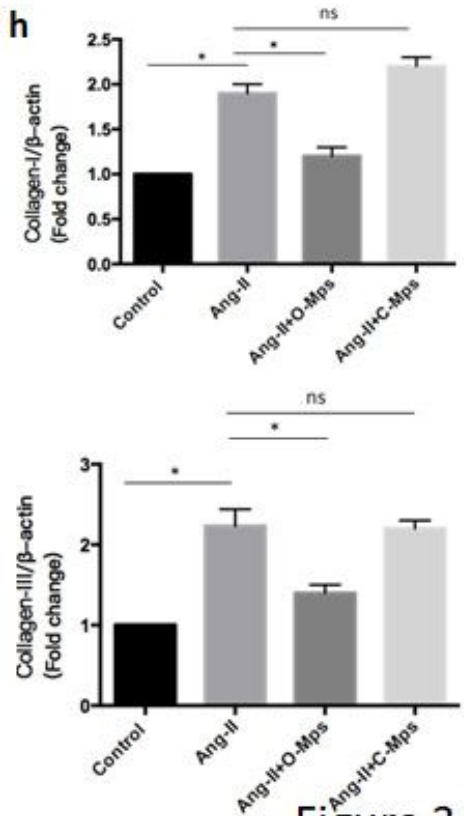

Figure 3

Figure 3 
O-MPs inhibited the proliferation, migration, and fibroblast-to-myofibroblast transition of cardiac fibroblasts. Two to three generations of neonatal rat cardiac fibroblasts were used in the experiment. (a) MTT assay, and (b) BrdU experiment showed the proliferation of cardiac fibroblasts. (c, d) Transwell experiments showed the migration of cardiac fibroblasts. Western blot was used to detect expression of $(e, f)$ a-SMA and $(g, h)$ type 1 and type 3 collagens. (i) Immunofluorescence in cardiac fibroblasts. F-actin was stained by FITC-labeled phalloidin and nuclei were stained with DAPI. Less F-actin and microfilament structure were observed in the O-MP group compared with the Ang-ll group. (j) Content of CTGF and TGF$\beta 1$ in heart tissue by ELISA. Data were obtained from at least three independent experiments and results are means \pm SD; Student's t-test, *P $<0.05$ vs. control or Ang-II. 

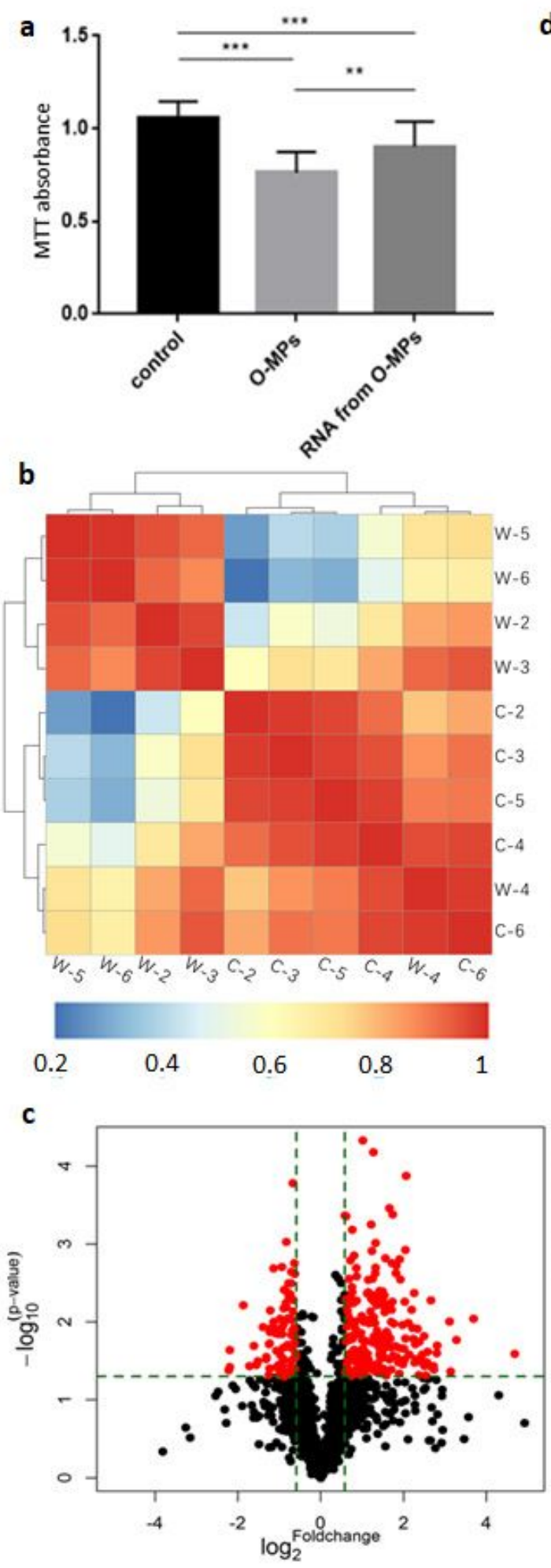

d

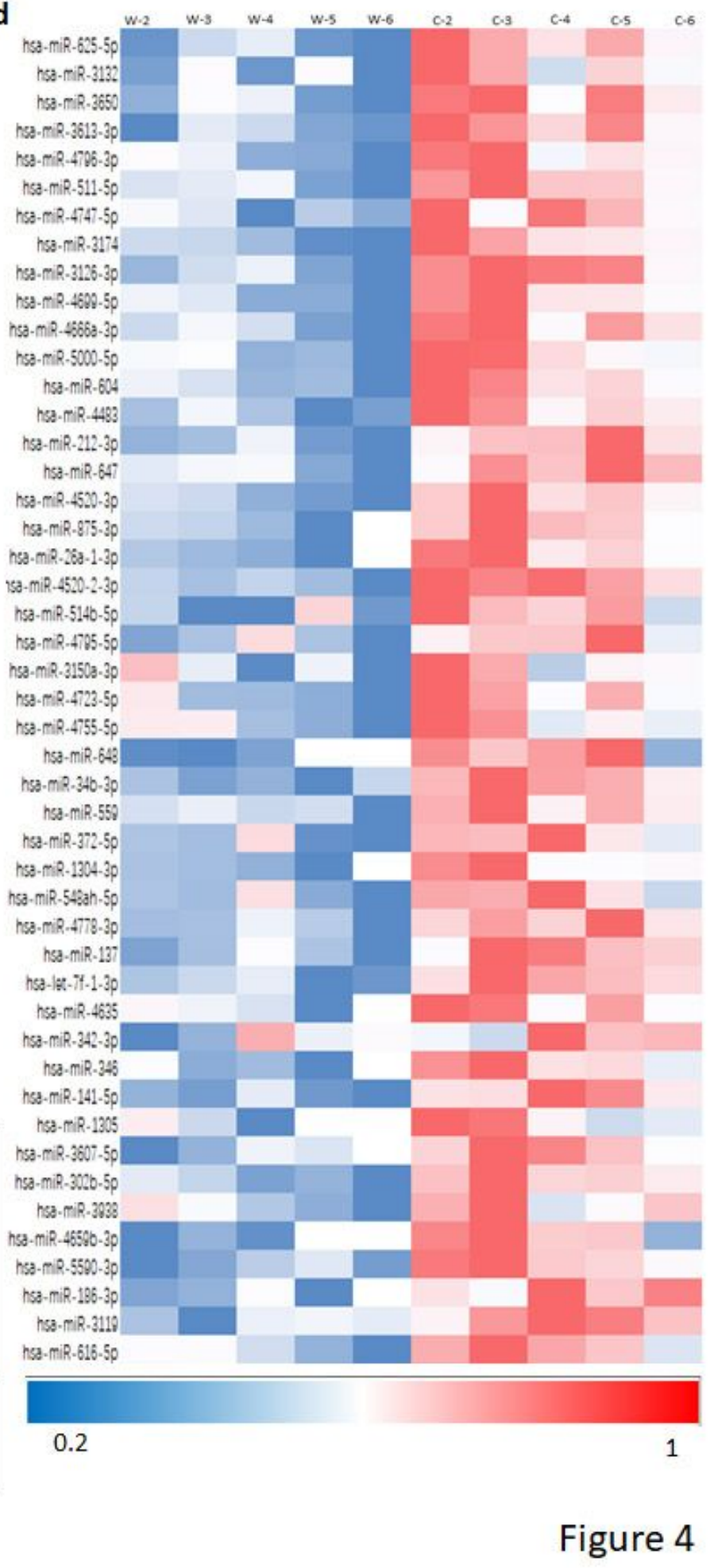

\section{Figure 4}

RNA microarray analysis of MPs. (a) MTT experiments showed the proliferation of cardiac fibroblasts after treatment with miRNAs from O-MPs. Data were obtained from at least three independent experiments and results are means \pm SD; Student's t-test, ${ }^{\star *} \mathrm{P}<0.01$, ${ }^{\star \star *} \mathrm{P}<0.001$ vs. control or 0 -MPs. (b) Pearson's correlation among biological replicates for all samples. (c) Volcano plot showing up- and 
downregulated miRNAs in O-MPs compared with C-MPs. (d) Heatmap of 47 upregulated miRNAs that might regulate TGF- $\beta 1$ and CTGF.
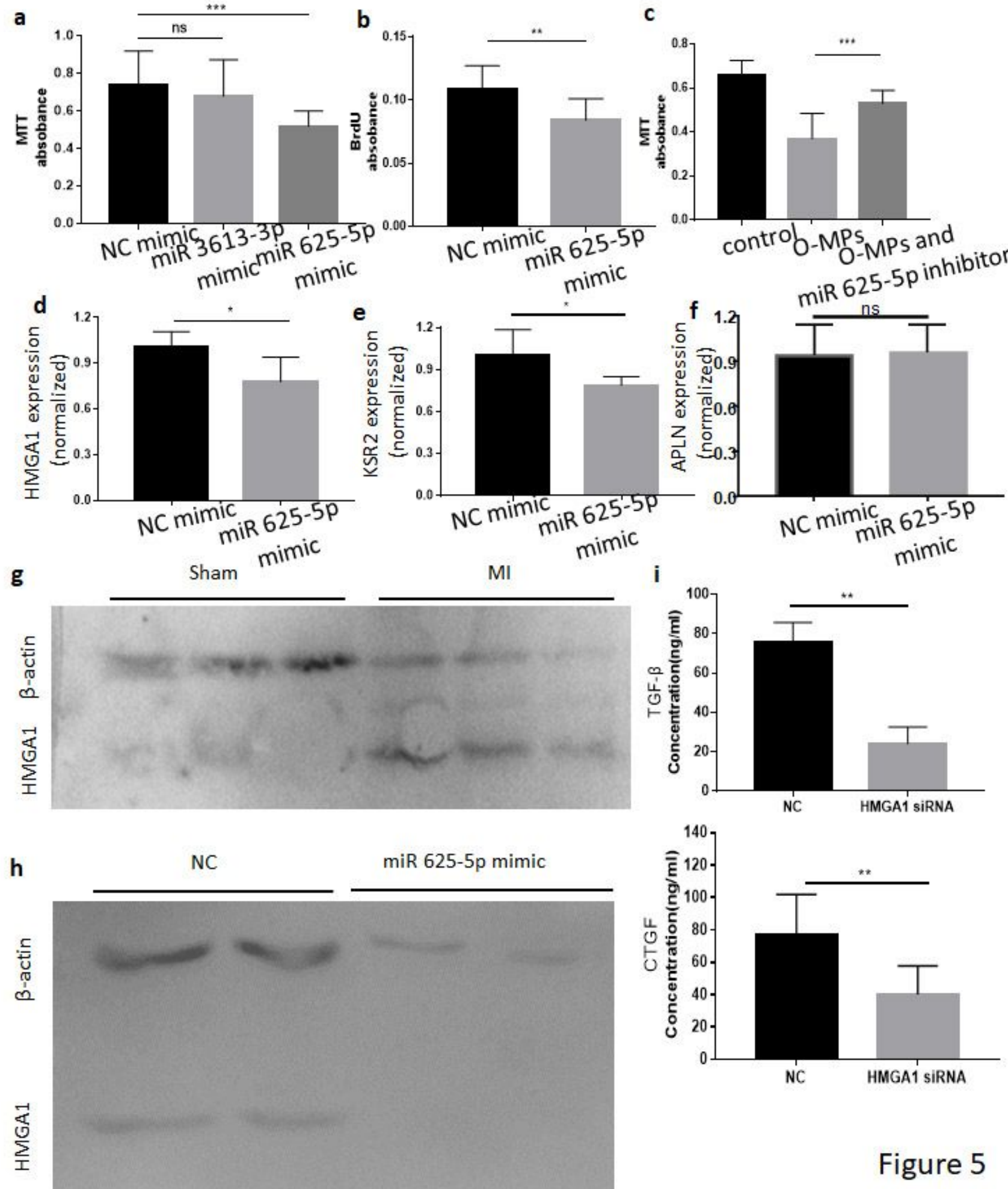

Figure 5

\section{Figure 5}

miR-625-5p inhibits proliferation of human cardiac fibroblasts (HCF) in vitro. (a) MTT assay and (b) BrdU experiment showed the proliferation of human cardiac fibroblasts after treatment with miR-625-5p or miR-3613-3p mimics. (c) MTT assay after treatment with miR-625-5p inhibitor and 0-MPs. (d, e, f) Real- 
time PCR of potential targets of miR-625-5p. (g) Western blot of HMGA1 in heart tissue after MI. (h) Western blot of HMGA1 in HCF after treatment with miR-625-5p mimic. (i) ELISA of TGF- $\beta 1$ and CTGF levels after treatment with HMGA1 siRNA. Data were obtained from at least three independent experiments and results are means \pm SD; Student's t-test, ${ }^{*} P<0.05,{ }^{*} \mathrm{P}<0.01,{ }^{*} * \mathrm{P}<0.001$ vs. control.

a

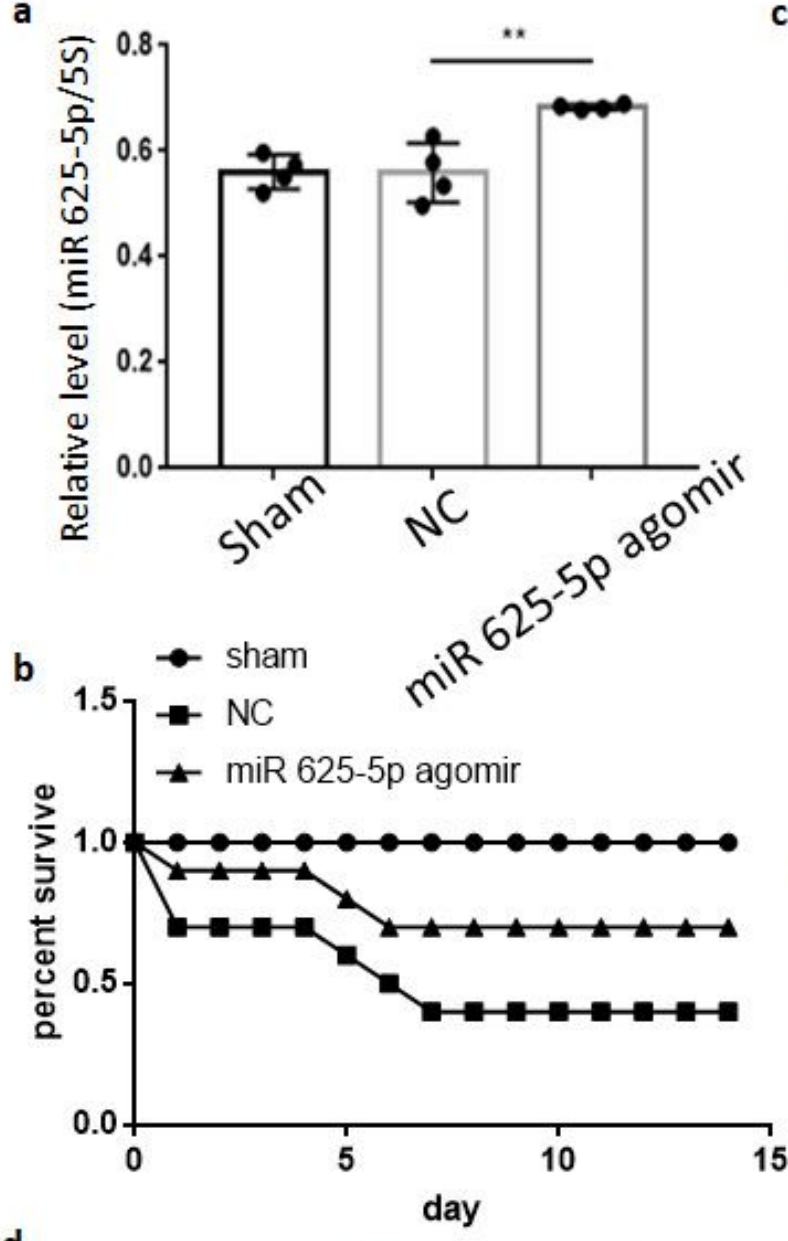

d

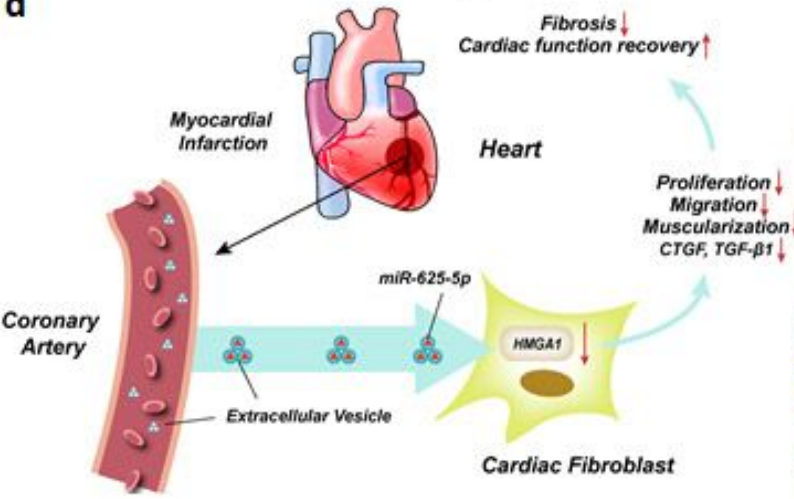

c
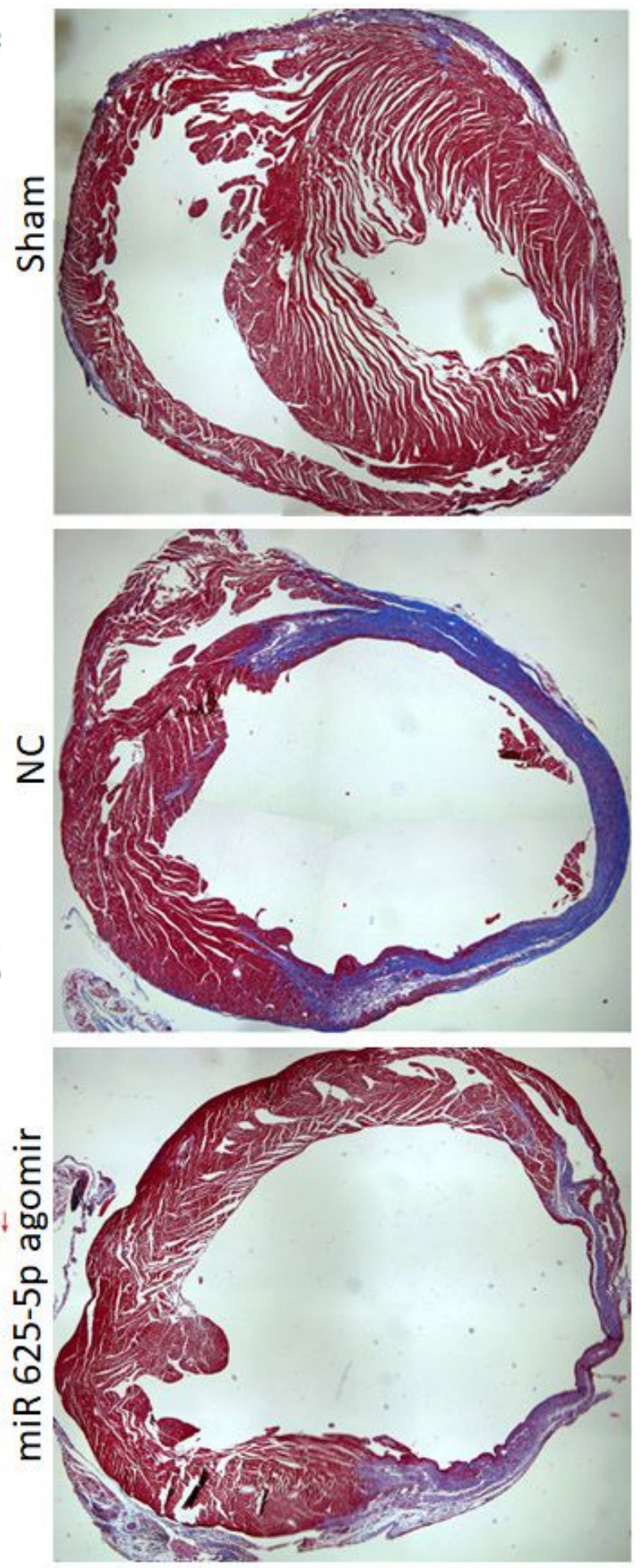

Figure 6

Figure 6 
miR-625-5p inhibits myocardial fibrosis after MI in vivo. (a) RT-PCR quantification of miR-625-5p levels in heart tissue 14 days after surgery and intramyocardial injection. Each group $n=4$, results are means $\pm S D$; Student's t-test, ${ }^{* *} \mathrm{P}<0.01$ vs. NC. (b) Survival curve of mice after Ml; sham group, $n=6 ; N C$ control group, $n=10 ;$ miR-625-5p agomir group, $n=10$. (c) Masson trichrome staining of hearts of mice showed collagen deposition 14 days after surgery. (d) After MI, MPs containing miR-625-5p in coronary artery acted on HMGA1 on fibroblasts, thereby reduced myocardial fibrosis. 